\title{
Rhabdoid Signet- Ring Mixed Cell Subtype Gastrointestinal Stromal Tumor from the Stomach - Pathohistological and Immunohistochemical Analysis, Differential Diagnosis, Prognosis and Complex Treatment
}

\author{
Lena Marinova ${ }^{*}$, Bistra Yordanov ${ }^{2}$, Ruslan Kulinski ${ }^{3}$, Nikolay Evgeniev ${ }^{4}$ and Radoslav Georgiev ${ }^{5}$ \\ ${ }^{1}$ Department of Radiotherapy, Complex Oncology Center, Bulgaria
}

${ }^{2}$ Department of Clinical Pathology, Complex Oncology Center, Bulgaria

${ }^{3}$ Department of Surgery, Complex Oncology Center, Bulgaria

${ }^{4}$ Department of Medical Oncology, Complex Oncology Center, Bulgaria

${ }^{5}$ Department of Imaging, Radiation therapy and Nuclear medicine, UMHAT"St. Marina", Bulgaria

*Corresponding author: Lena Marinova, Department of Radiotherapy, Complex Oncology Center, Ruse, Bulgaria

To Cite This Article: Lena Marinova, Bistra Yordanov, Ruslan Kulinski, Nikolay Evgeniev, Radoslav Georgiev. Rhabdoid Signet- Ring Mixed Cell Subtype Gastrointestinal Stromal Tumor from the Stomach - Pathohistological and Immunohistochemical Analysis, Differential Diagnosis, Prognosis and Complex Treatment. Am J Biomed Sci \& Res. 2021 - 13(3). AJBSR.MS.ID.001867. D0I: 10.34297/AJBSR.2021.13.001867.

Received: 眥 May 05, 2021; Published: 制 June 25, 2021

\begin{abstract}
We present rarely diagnosed extremely malignant rhabdoid signet- ring mixed cell subtype gastrointestinal stromal tumor (GIST) from the stomach in a man of 67 years. This is a third rhabdoid histological phenotype GIST posted in the English medical literature. The diagnosis is placed after a pathochistological and immunohistochemical analysis of the lung biopsy material. The purpose of this presentation is to discuss the difficulty early diagnostics, the prognosis of this extremely malignant GIST, the differential pathohistological and immunohistochemical diagnosis with other benign and malignant tumors. The diagnosis of a GISTs is directly related to treatment with a tyrosine kinase inhibitor (Imatinib), which is indicated for inoperable and metastatic GIST.
\end{abstract}

Keywords: Gastrointestinal stromal tumor; Immunohistochemical analysis; Rhabdoid histologic phenotype GIST; Signet- ring cell GIST; Differential diagnosis; Complex treatment

\section{Introduction}

Gastrointestinal stromal tumor (GIST) is the most common mesenchymal tumor of gastrointestinal tract [1,2], although GIST may arise from any portion of the foregut to hindgut, two thirds of stromal tumors originate from the stomach [3]. GISTs are thought to arise from the interstitial cells of Cajal (ICC) [4]. Their functions include generation of pacemaker activity and inhibitory neurotransmission [5-7]. ICC normally express cKIT (CD 117), which is a tyrosine kinase growth factor receptor. This cKIT immunoreactivity is the best defining feature of GISTs, distinguishing them from true smooth muscle tumors (i.e., leiomyomas and leiomyosarcomas) and tumors arising from neural crest derivatives (i.e., schwannomas and neurofibromas) [8]. According to Fletcher et al. [9], GISTs can be divided into categories based on size and mitoses: very low risk, low risk, intermediate risk, and high risk. We present a clinical case with a extremely malignant rhabdoid Signet- ring cell mixed type gastrointestinal stromal tumor (GIST) from the stomach with extremely quickly distant metastases to determine the importance of immunohistochemical 
analysis defining differential diagnosis, prognosis and the need for complex treatment.

\section{Clinical Case}

We present a 67-year-old patient with initial bulk complaints in the chest and left lumbar with constant pain and purse secretion.

\section{Objective-local status}

Left breast-lumbar area- Swelling, redness, local hyperthermia with blood, pus, and other secretions.

Lung - two-sided purely vesicular breathing without wheezing From paraclinical studies on 18.02.21, accelerated sludge in the blood - $88 \mathrm{~mm} / \mathrm{h}$; leukocytosis - 17.1 WBCs per microliter of blood; Hemoglobin-110 gm/dL; Common serum protein -72.5 g/dl; C-reactive protein (CRP)-10,4697 mg/L.

\section{CT with venous contrast, mediastinal and pulmonary window}

Double-sided pleural effusions, larger left, with some compression on the ninth and tenth segment of the left lower lung lob. A parenchyma solid contrast-amplifying lesion, most likely metastatic, is visualized peripherally in the fourth segment to the left, close to the chest wall. Mediastinal lymphadenopathy (Figure 1/A); Pulmonary CT scan after 1 month - slightly increased pleural effusion on the left, as well as parenchymal pulmonary lesion on the left associated with the chest wall (Figure 1/B).

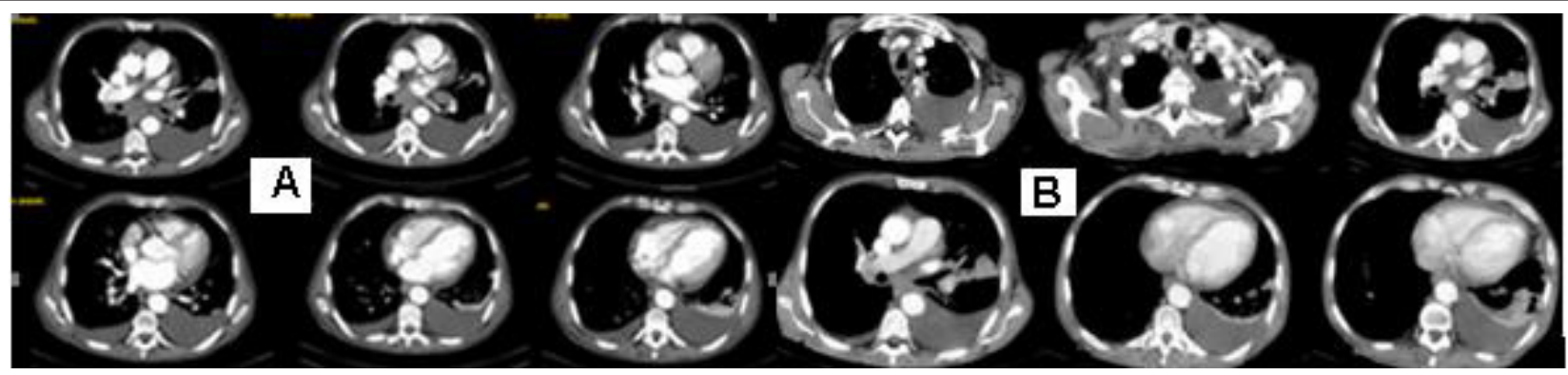

Figure 1: CT with venous contrast, mediastinal and pulmonary window: A/double-sided pleural effusions, larger left, with some compression on the ninth and tenth segment of the left lower lung lob. A parenchyma solid contrast-amplifying lesion is visualized peripherally in the fourth segment to the left, close to the chest wall. Mediastinal lymphadenopathy. B/ Pulmonary CT scan after 1 month without substantial dynamics.

\section{Abdominal CT}

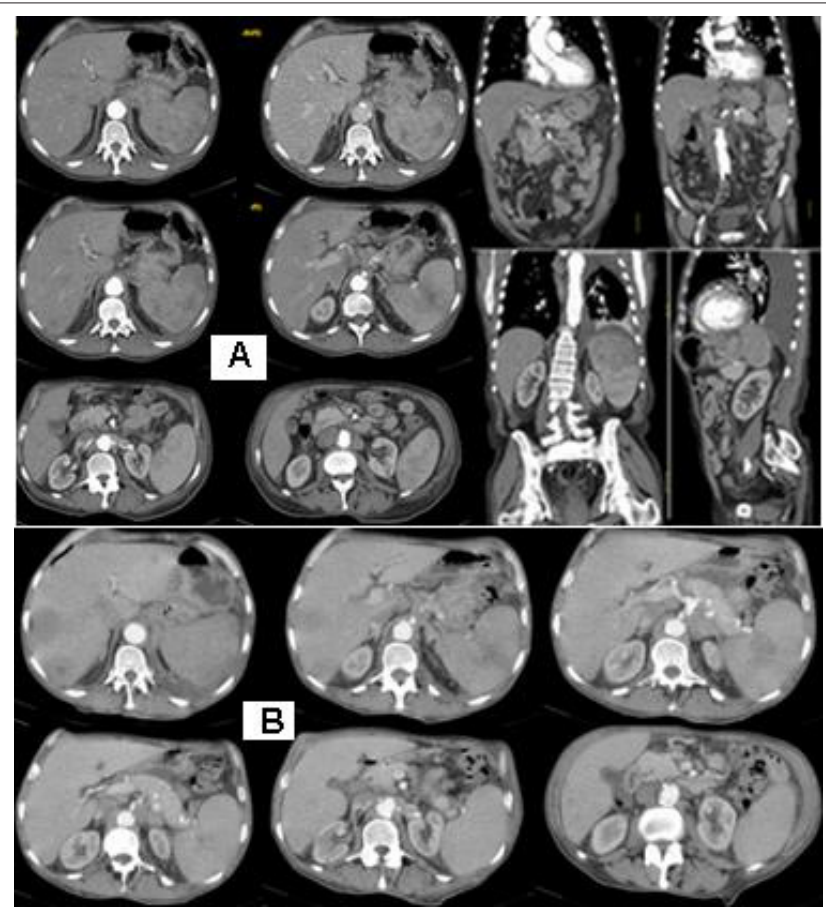

Figure 2: Abdominal CT with venous contrast, with axial transverse images and sagittal and coronal reconstructions: $\mathrm{A} / \mathrm{A}$ parenchymal mass lesion, originating in the stomach, with exophytic growth and incidental infiltrations, which has increased dimensions changed spotted hypodense structure, and pushes the left kidney medially and down. The formation structure is inhomogeneous, with a soft density, with the presence of hypodense areas centrally, possibly necrotic. Expressed lymphadenopathy paraaortal, around the stomach and the Celial Trunkus; B/ Abdominal CT with axial transverse images after 1 month with visible liver metastases and increased gastric formation with local infiltration in the spleen. 
Abdominal CT with venous contrast, with axial transverse images and sagittal and coronal reconstructions: a parenchymal mass lesion, originating in the stomach, with exophytic growth and incidental infiltrations, which has increased dimensions changed spotted hypodense structure, and pushes the left kidney medially and down. The structure of the formation is inhomogeneous, with a soft density, with the presence of hypodense areas centrally, possibly necrotic. Expressed lymphadenopathy paraaortal, around the stomach and the truncus coeliacus (Figure 2/A). Abdominal CT scan after 1 month with visible liver metastases (Figure 2/B).

\section{Conclusion from Imaging Diagnostics}

Most likely concerns pulmonary carcinoma on the left with engaging mediastalline lymphatic chains, engaging abdominal and mesenteric lymph nodes. A parenchymal mass lesion, originating in the stomach with infiltration of the spleen and gastric fundus is detected. Gastroscopy with biopsy is recommended.

\section{From the endoscopic biopsy of the stomach mucosa}

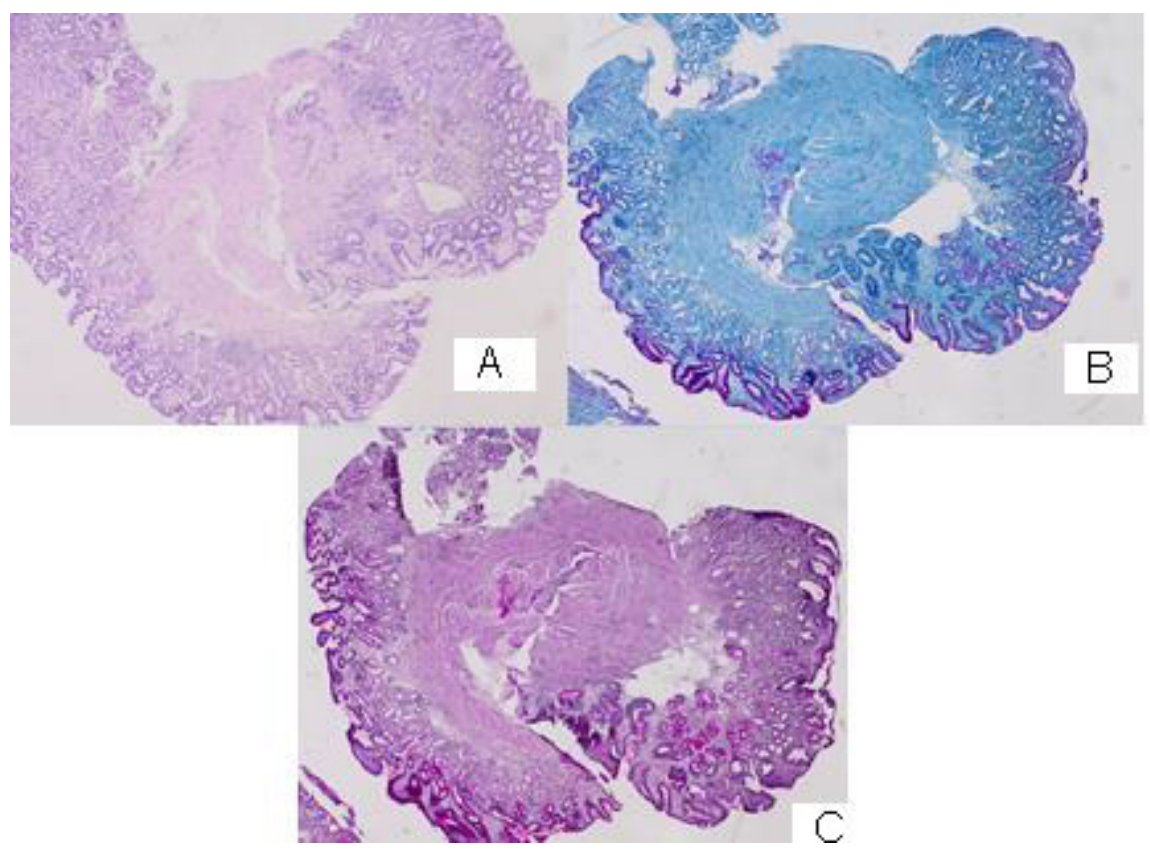

Figure 3: Photomicrography from the endoscopic biopsy of the stomach mucosa: A/ atrophic gastritis without a Helicobacter H\&E: 20x; B/ Atrophic gastritis and Paneth cell metaplasia (PAS -Alcian blue): 20x; C/ Atrophic gastritis and Paneth cell metaplasia (PAS): 20x.

There is a slightly pronounced cell dysplasia in single glands, interstitial and Paneth cell metaplasia, hypertrophy of Muscularis Mucosae with chronically detected inflammation, corresponding to atrophic gastritis without Helicobacter pylori (Figure 3/A,B,C).

\section{Surgical intervention}

An incision on the flender of the left chest followed by fasciotomy with necrectomy and exudat evacuation. Under CT control a percutaneous trans-cut biopsy from the left lung lesion is carried out.

\section{Histological Result}

Tissue cylinders from lung biopsy composed of fusiform atypical cells with hyperchromic nuclei with characteristic perivascular arrangement and the presence of vorticity cellular pattern. Tumor cells are located among eosinophil hyalin-like stroma with the presence of intercytoplasmic vacuols and perinuclear halo, so most of them have signet-ring cell views. Tumor cells tightly infiltrate thin-walled vessels and places entering the lumen. In the lesion, thick-walled arterioles are also found. Tumor nuclei are polymorphs with visible nucleolus, cigar-shaped and blunt-ended with variable atypia, often with cytoplasmic vacuoles at both ends of nuclei (Figure 4).

\section{Immunohistochemical (IHC) analysis from transthoracic lung biopsy}

Positive expression for CD 117 in numerous rhabdoid tumor cells, showing strong cytoplasmic staining; Positive IHC expression for CD 34 in single tumor cells with large round or fusiform nuclei, and the blood vessels forming structures with the type of deer horns; Diffuse positive expression for SMA in spindle tumor cells with elongated, blunt-ended, segmented or fusiform nuclei, but not in epitheloid cells; Positive IHC expression for TFT1 in single tumor cells with big rounded nuclei; Diffuse positive expression for Vimentin in tumor cells (Figure 5-8C/D). The tumor population indicates negative expression for CDX2; CK 20; CK AE1-AE3; Calretinin and S-100 Protein; Ki 67-40.39\%. 


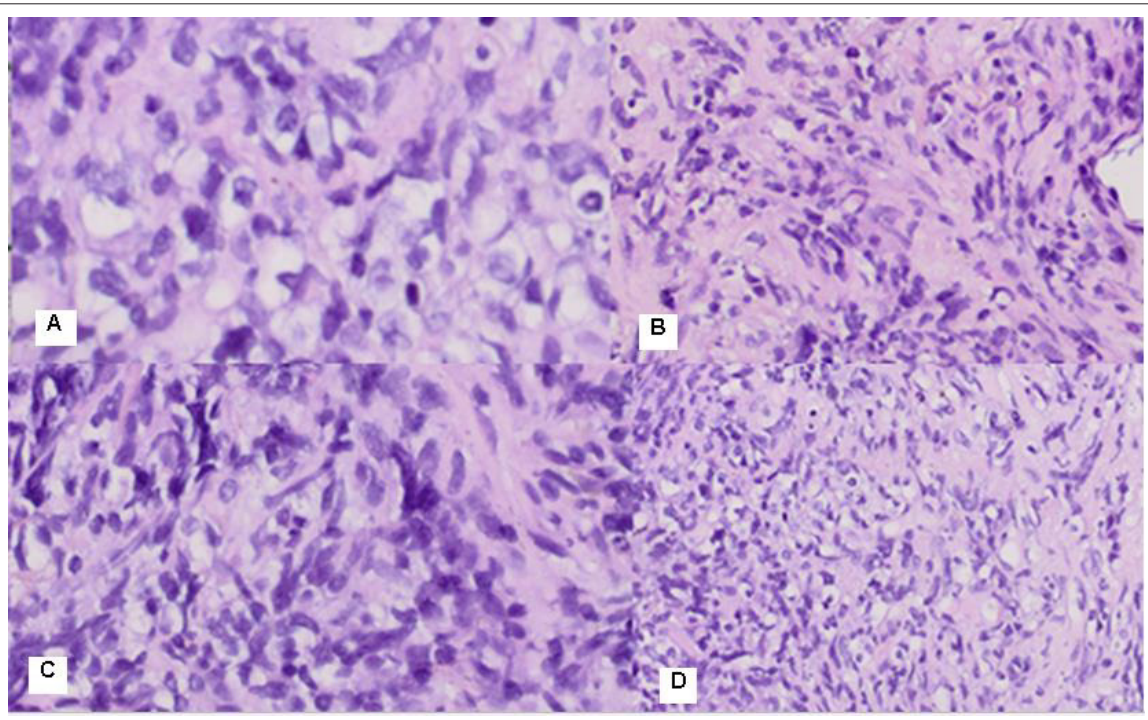

Figure 4: Photomicrography of pulmonary biopsy composed of fusiform atypical cells with hyperchromic nuclei with perivascular arrangement and the presence of vorticity cellular pattern. Cells are located among eosinophil hyalin-like stroma with the presence of intercytoplasmic vacuols, so most of them have signet-ring cell views. The most common cells were spindle cells with elongated, blunt-ended, segmented or fusiform nuclei, and round/polygonal cells, often with rounded or indented nuclei A/ H\&E: 400x; B/ H \&E: 40x; C/ H\&E:100x; D/ H\&E:20x.

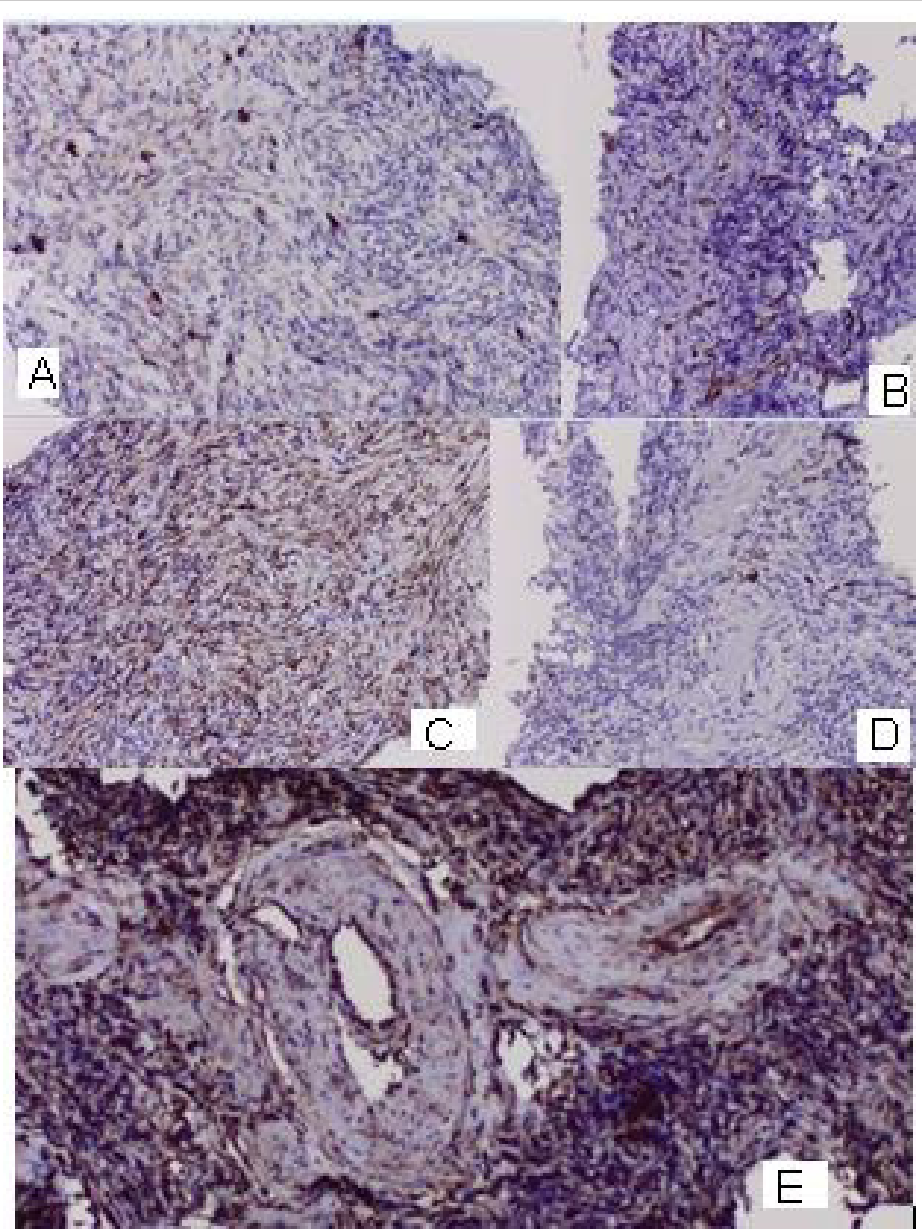

Figure 5: Photomicrography of immunohistochemical analysis from pulmonary biopsy: A/ Positive expression for CD 117 in numerous rhabdoid tumor cells, showing strong cytoplasmic staining; 20x; B / Positive IHC expression for CD 34 in single tumor cells with large round or fusiform nuclei and in numerous rhabdoid tumor cells of the tumor neoangiogenesis; 20x; C / Diffuse positive expression for SMA in tumor cells; 20x; D / Focal positive expression for TFT1 in a small fraction of tumor cells; 20x ; E/ Diffuse positive expression for Vimentin in tumor cells; 20x. 


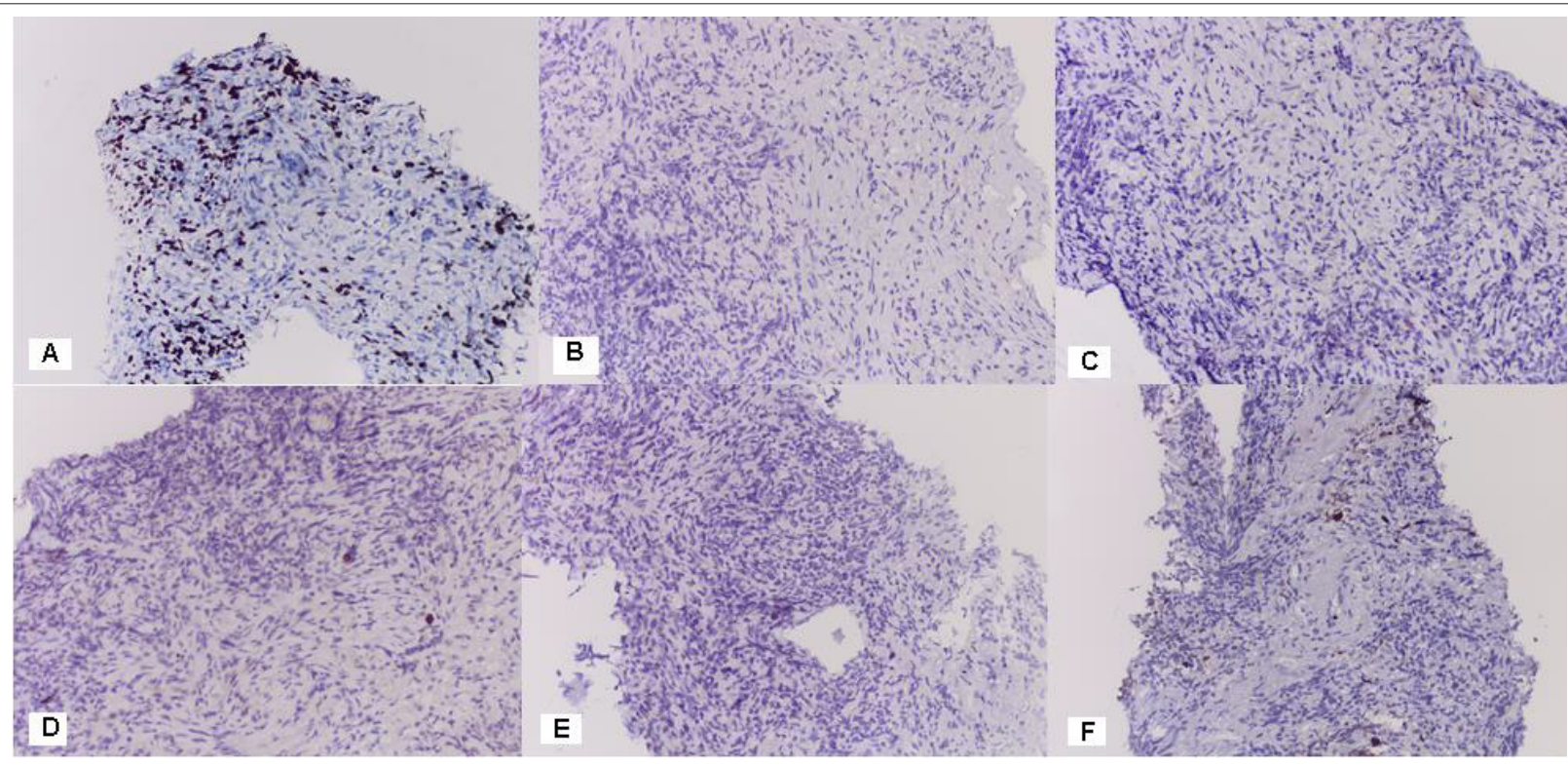

Figure 6: Photomicrography of immunohistochemical analysis from pulmonary biopsy: A / Ki $67-40.39 \%$ 20x; A tumor population indicates negative expression for: B / CDX2; C / CK 20; D / CK AE1-AE3; E / Calretinin; F / S-100 Protein (20x).

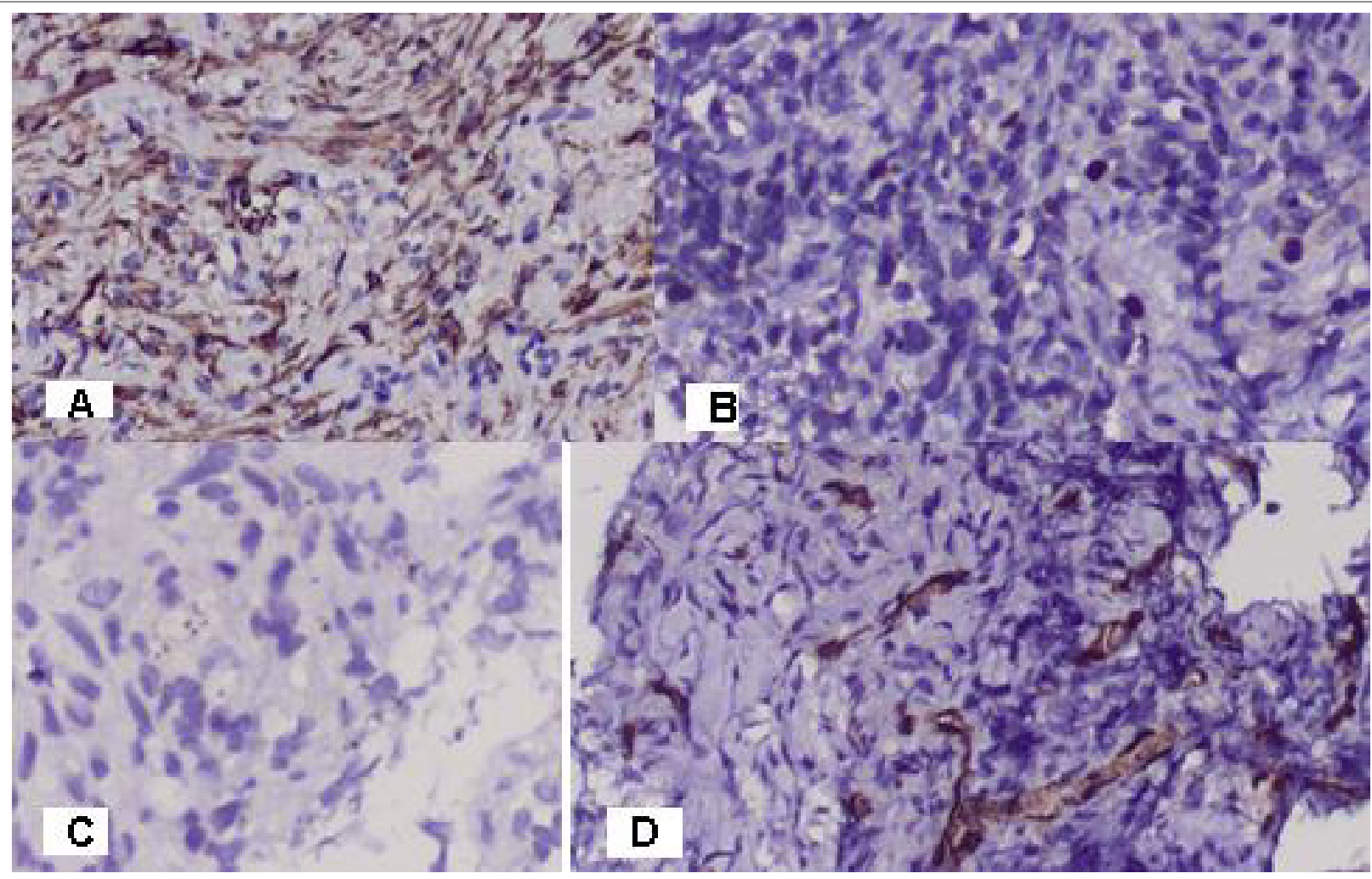

Figure 7: Photomicrography of immunohistochemical analysis from pulmonary biopsy: A/ Diffuse positive expression for SMA in spindle tumor cells with elongated, blunt-ended, segmented or fusiform nuclei, but not in epitheloid cells; $20 \mathrm{x}$. B/ Positive IHC expression for TFT1 in single tumor cells with big rounded nuclei; $20 \mathrm{x}$. C/ Immunohistochemical analysis with Calretinin has negative expression in tumor cells; 20x. D / Positive IHC expression for CD 34 in single tumor cells with large round or fusiform nuclei and in numerous rhabdoid tumor cells. Tumor blood vessels are type of deer horns, branched and rich blooded, enhancing distant blood metastases; 20x. 


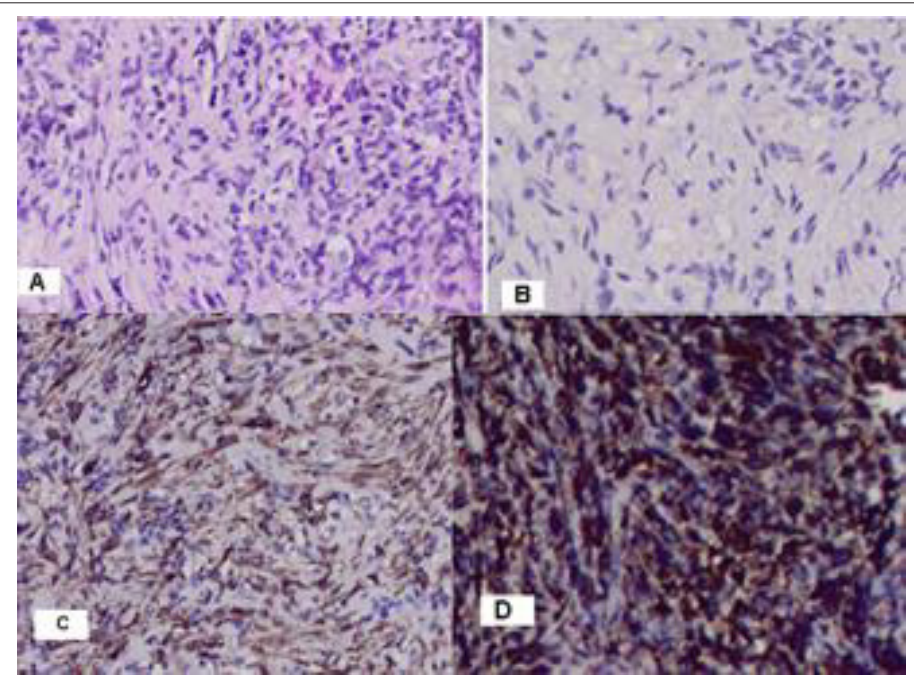

Figure 8: Photomicrography of pulmonary biopsy: A/ Tumor cells are located among eosinophil hyalin-like stroma with the presence of intercytoplasmic vacuols and perinuclear halo, so most of them have signet-ring cell views H\&E:20x; B/ Nuclei are cigar-shaped and blunt-ended with variable atypia, often with cytoplasmic vacuoles at both ends of nuclei H\&E:20x; C/ DD with spindle cell liposarcoma, whose tumor cells do not express SMA; 20x; D/ Highly diffuse positive expression for Vimentin in all tumor cells; 20x.

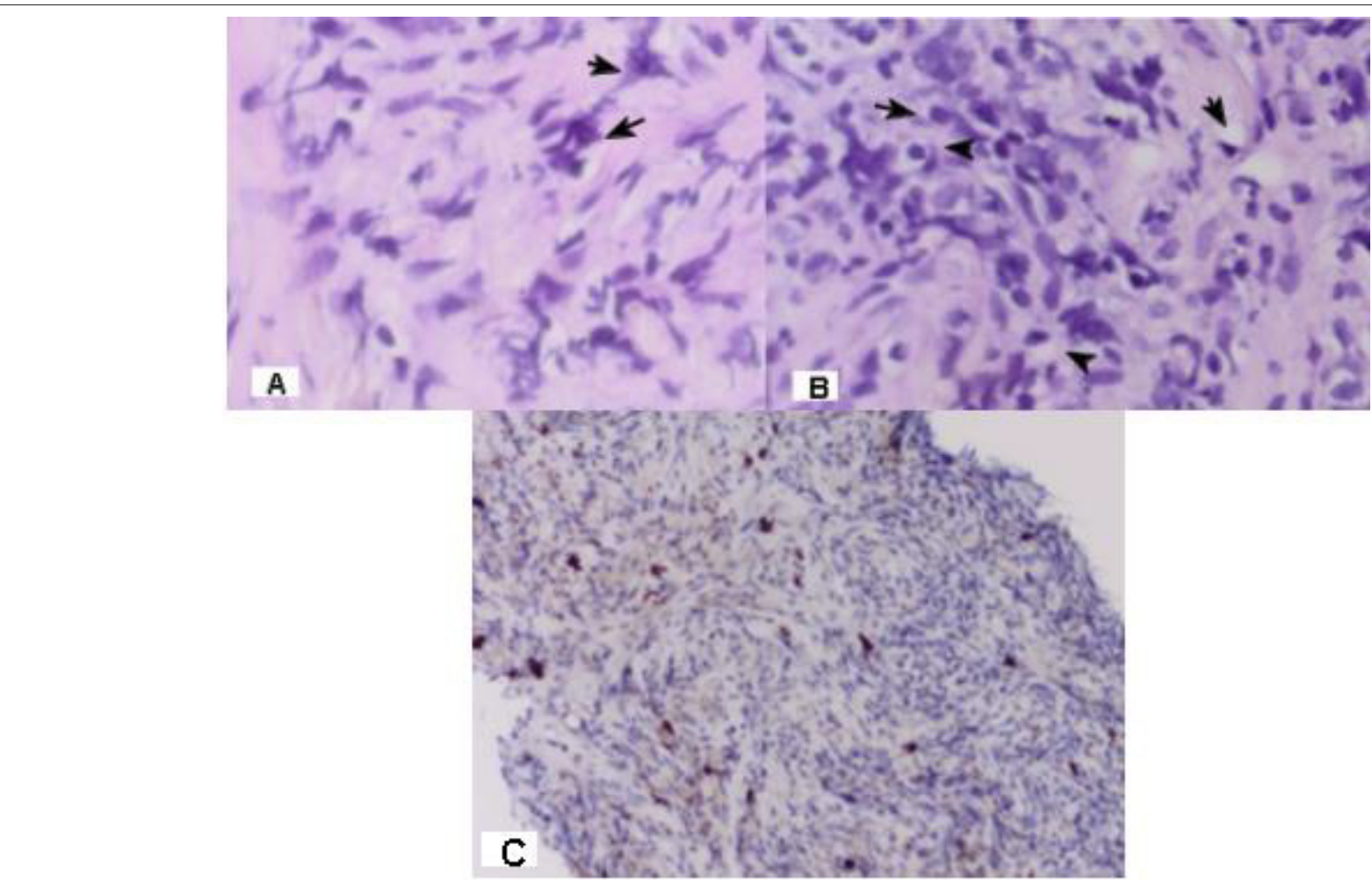

Figure 9: Photomicrography of pulmonary biopsy; A/ With the black arrows are shown rabdoid tumor cells; $400 \mathrm{x}$; B/ With the black arrows are shown signet-ring tumor cells; H\&E: 400 x; C/ Positive IHC expression for CD 117 in numerous rhabdoid tumor cells; 20x.

Final histological diagnosis after extensive pathochistological and immunohistochemical analysis: Rhabdoid signet- ring mixed cell (spindle and epithelioid) subtype GIST.

The imaging diagnostics reports local GIST infiltration in spleen with metastases in the lung, liver, mediastinal, abdominal and mesenteric lymph nodes and subcutaneous metastasis.
The patient was targeted for specific drug treatment with a tyrosine kinase inhibitor (i.e. Imatinib), which is indicated for inoperable and metastatic GIST.

\section{Discussion}

In 2004, Huang XF et al. [10] express an opinion that Leiomyoblastoma, also called bizarre leiomyoma or epithelioid 
leiomyoma, is a rare smooth muscle tumor characterized by epithelioid cells with clear cytoplasms and an unknown biological behaviour. Leiomyoblastoma is a rare, smooth muscle tumor of the stomach that occurs chiefly in the antrum [11]. For decades it was thought that the majority of gastrointestinal mesenchymal tumors arose from the smooth muscle, being called "leiomyomas" and "leiomyosarcomas" [12]. Gastric leiomyoblastoma of Martin's myoid tumour or Stout's smooth muscle cell bizarre tumour is a rare benign mesenchymal neoplasia, that may become malignant $[13,14]$. Leiomyoblastoma and gastrointestinal autonomic nerve tumor (GANT) are old terms no longer used. The new terminology for these tumors is Gastrointestinal stromal tumor (GISTs) [15]. Approximately 60 to $70 \%$ of GISTs arise in the stomach, 20 to $30 \%$ in the small intestine, $5 \%$ in the colon and rectum [4]. The discovery that GISTs could express KIT protein helps establish that GISTs do not originate from smooth muscles. The current thinking is that GIST tumors arise either from stem cells that differentiate towards interstitial cells of Cajal or directly from interstitial cells of Cajal (ICCs) [16]. Only recently it was clarified that this neoplasm is a well-defined disease called GIST, the acronym for gastrointestinal stromal tumor, through the discoveries of its origin from the interstitial cells of Cajal [17] and the expression of c-Kit protein [18].

\section{Diagnosis}

Leiomyoblastoma (GIST) is a rare smooth muscle tumor characterized by epithelioid cells with clear cytoplasms and an unknown biological behaviour. Since pre-operative diagnosis is difficult, the optimum strategy during the operation could be determined onlybyhaving athorough knowledge aboutitbeforehand [19]. The most important CT findings for diagnosis of gastric GIST are: location in the body/fundus, predominantly extraluminal growth, peripheral hypervascularity with heterogeneous core [20]. Because most GISTs show an exophytic growth pattern and arise within the muscularis propria of the stomach or intestinal wall, they present as dominant masses outside of the organ of origin [21]. In early disease, the lesion will remain located subepithelially, therefore, the conventional endoscopic biopsy is of value only when positive, since the tumor may be situated deeper. In the endoscopy unit of INCA, the technique was developed to remedy this situation in addition to endoscopic ultrasound [12]. In general, GISTs are characterized by a uniform, monotonous appearance with minimal cytologic atypia or mitotic activity. Nuclear pleomorphism is occasionally evident in a GIST and, when present, is often admixed with the more conventional cytologic features [22]. Many malignant GISTs have areas that appear benign, such that sampling can be a huge problem. It is also conceivable that the area biopsied could be CD117 negative whereas other parts of the tumor are positive, leading to a completely erroneous diagnosis [23]. In our clinical case in the material from the stomach biopsy, we found a slightly pronounced cell dysplasia in single glands, interstitial and Paneth cell metaplasia, hypertrophy of muscularis mucosae with chronically detected inflammation, corresponding to atrophic gastritis without Helicobacter pylori. Paneth cell metaplasia is abnormal and is considered a sign of long-term damage to the tissue and is an indicator of chronic epithelial cell damage [24]. In the clinical case, the endoscopic biopsy of the stomach mucosa is negative. as it is from the stomach mucosa rather than the muscle layer in which the tumor formation expanded (Figure 3). Due to a severe general condition of the patient at a high risk of surgery (gastrectomy), the diagnosis we achieved after a pathochistological and immunohistochemical analysis of the lung biopsy material.

\section{Biological Development}

Gastrointestinal stromal tumors (GISTs), the most common mesenchymal tumors in the gastrointestinal tract, form a specific problem as they have the ability to metastasize, even though they may appear to be fully benign [9,25]. GIST often spreads from the original (primary) site to distant locations. If GIST tumors metastasize they usually travel to the liver, or the peritoneum. Metastases to lymph-nodes and lungs are rare but do occur [16]. A case of recurring GIST of the stomach that metastasized to the spleen and the liver has been published [26]. An interesting pathologist structure we observe in our clinical case is the presence of vorticity cellular pattern (Figure 4/B,C,D and Figure 5/E), which is characteristic of the division of endothelial cells. These cellular divisions induce long-range, well-ordered vortex patterns extending several cell diameters away from the division site [27]. The process of endothelial cell division is essential for angiogenesis and vessel repair [28], as well as for the growth of metastasis from malignant tissue [29]. Tumor vascularization may be a result of a few different potential mechanisms. Apart from angiogenesis, cancer may achieve new vasculature by vessel co-option (using existing vessels), vascular mimicry (the process of forming vessels from tumor cells) and vasculogenesis (involving bone marrow-derived progenitor cells) [30]. In our clinical case, there is no symptoms on the side of the stomach from where the tumor formation originates. The initial clinical expression of the tumor is by the soft and lung metastasis, followed by liver metastases. In Figure 7/D there is a rich tumor neoangiogenesis, which is one of the causes of rapid hematogenic desimination of a GIST. Tumor blood vessels are type of deer horns, branched and rich blooded, enhancing distant blood metastases. We present an extremely aggressive vascular mimicry GIST, which is manifested by its distant blood metastases in lung, liver and lymph nodes (Figure 1A/B, Figure 2A/B).

\section{Prognosis}

A panel of five criteria (tumor size, mitotic rate, proliferation index, presence of necrosis, and invasion of mucosa or adjacent structures) have been devolped and that permitted distinction 
between low and high malignant potential GISTs [31]. They are rare disease, however up to $2 / 3$ developed recurrence or metastasis during the follow up. This risk is higher in high potential malignant tumors and the study of tecidual markers as Ki67 may help to decide which patient will have worst prognosis [32]. Mitotic index is by far the most important prognostic feature of GISTs [33]. The literature articles related that up to $20 \%$ of GISTs tumors had a high malignant potential $[34,35]$, however this potential arise to $80 \%$ [32]. In our clinical case, Ki 67 is quite high (40,39\%), which determines the high mitotic activity of tumor cells and their malignant potential (Figure 6/A). Some experts feel that all stromal tumors are potentially malignant and hence that tumors should be classified as low risk and high risk, whereas others prefer not to mention prognosis at all [23].

\section{Histopathological Analysis}

Microscopically, most GISTs demonstrate three main histologic subtypes: spindle cell type (most common), epithelioid type, and mixed spindle and epithelioid type $[2,9,35]$. The mixed type GISTs is the most rarely diagnosed, only $10 \%$ of these stromal mesenchymal tumors [36]. Malignant spindle cell gastric stromal tumors tend to lose perinuclear vacuoles. Another curious pattern of growth in gastric stromal tumors is the epithelioid variant (Stout's leiomyoblastoma) [23]. Epithelioid GISTs account for approximately $20 \%$ of cases and are characterized by rounded cells arranged in nests or sheets, with variably eosinophilic to clear cytoplasm and vesicular nuclei [9,37]. These tumors are composed of plump or rounded cells, many of which show marked nuclear pleomorphism. GISTs are KIT-positive spindle cell, epithelioid, or rarely pleomorphic mesenchymal tumors with characteristic histologic features and occurring anywhere in the gastrointestinal tract or abdomen [38]. CD 117 is considered the most specific criteria for the diagnosis of GIST [8]. In Figure 9A/B/C establish rabdoid cells and resembling signet- ring cells, which determines that it is about a clinical case with rhabdoid signetring mixed (spindle and epithelioid) cell subtype GIST. Epithelioid and fusiform atypical cells with hyperchromic nuclei are located among eosinophil hyalin-like stroma. The most common cells were spindle cells with elongated, blunt-ended, segmented or fusiform nuclei, and round/polygonal cells, often with rounded or indented nuclei. A characteristic feature is the presence of intercytoplasmic vacuols in tumor cells and perinuclear halo, so most of them have signet-ring cell views. Tumor nuclei are polymorphs with visible nucleolus, cigar-shaped and blunt-ended with variable atypia, often with cytoplasmic vacuoles at both ends of nuclei (Figure 4 and Figure 8A/B).

\section{Differential Diagnosis- Leiomyosarcoma (LMS)}

It can be categorized into uterine, retroperitoneal, nonretroperitoneal soft tissue, cutaneous, visceral, and osseous anatomic subtypes [39]. LMS is a smooth muscle tumor with atypia plus either mitotic activity, tumor cell necrosis or size $>10 \mathrm{~cm}$. Tumor cells merge with blood vessel walls. LMS is constructed from palisading of spindle cells with eosinophilic fibrillary cytoplasm, focal granularity. Nuclei are cigar-shaped and blunt-ended with variable atypia, often with cytoplasmic vacuoles at both ends of nuclei (unlike neural lesions) May have hemangiopericytoma-like vasculature change [40]. The most common cells were spindle cells with elongated, blunt-ended, segmented or fusiform nuclei, and round/polygonal cells, often with rounded or indented nuclei [41]. Microscopically, the tumor cells are spindle-shaped with abundant eosinophilic cytoplasm and elongated nuclei [42,43]. Is reported marked atypia and necrosis; positive IHC for smooth muscle actin or desmin; negative for CD117; no KIT mutations [15]. Most tumors previously diagnosed as gastrointestinal autonomic nerve tumors (GANTs) are now classified as GISTs and contain essentially the identical KIT mutations as GIST [10]. Epithelioid sarcoma (ES) is a rare soft tissue sarcoma arising from mesenchymal tissue and characterized by epithelioid-like features. It accounts for less than 1\% of all soft tissue sarcomas and was first clearly characterized by F.M. Enzinger in 1970 [44]. ESs typically express vimentin, cytokeratins, epithelial membrane antigen, and CD34, whereas they are usually negative for S100, desmin, and FLI1 (FLI-1) [45]. ES demonstrates lymphatic spread (in 22-48\% of cases), and metastasis (in 21-63\% of cases) [46]. High-grade spindle cell sarcomas are rare, undifferentiated, pleomorphic sarcomas previously known as malignant fibrous histiocytomas that are rarely found, and present difficult diagnostic and treatment options in clinical practice [47]. Spindle cell liposarcoma (SCLS) is a rare variant of well differentiated liposarcoma characterized by prominent spindle cell component. Previously reported cases originated in the subcutaneous tissues of shoulder girdle and upper limb [13]. It is characterized that SCLS tumor cells are immunohistochemically positive for CD 34 and Vimentin with negative for Keratin and S100 protein, but not expressing SMA [48]. Inflammatory myofibroblastic tumor contains conspicuous inflammatory cells and can be positive for ALK1 and negative for CD117 [15].

\section{Immunohistochemistry (IHC)}

Before the advent of immunohistologic methods, most spindle cell sarcomas of the gastrointestinal tract were considered to be leiomyomas or leiomyosarcomas, with occasional examples of neurogenic tumors [8]. Gastrointestinal stromal tumor (GIST) is now defined as a specific, KIT-expressing and KIT-signaling driven mesenchymal tumor of the gastrointestinal (GI) tract [38]. GIST defines a distinct group of gastrointestinal tumors that originate from the intestinal cells of Cajal (ICCs) [8]. The ICCs are responsible for motility [49], show immunophenotypic and ultrastructural features of both smooth muscle and neural differentiation and 
express the Kit receptor (CD117) similar to GIST [12]. True GISTs can be identified from other tumors by histological analysis and the expression of KIT (CD117) [23], a tyrosine kinase inhibitor that is present in almost $95 \%$ of these tumors [50-52]. KIT is a transmembrane protein that stimulate the cell proliferation and inhibit the apoptosis [53,54]. There are rare CD117-negative GISTs but that such a diagnosis should probably only be rendered by an expert [23]. These are rare undifferentiated GIST with marked anaplasia and negative for CD117 [36]. Most GIST patients are CD34 and Vimentin positive [56], but negative for S-100 or desmin $[10,26,55,56]$. In cases where there is no certainty whether the lesion is a stromal tumor based on the H\&E-stained sections, we usually use CD34, S-100, and smooth muscle actin (SMA) (in addition to CD117) [23]. The tumors coexpressed CD34 and KIT and were rarely positive for SMA or desmin and never for S-100 protein [57]. Immunoreactivity for SMA is found in nearly $30 \%$ to $40 \%$ of GISTs [22].

In the presented clinical case, like Jeffrey AR et al. [58], both spindled and rhabdoid tumor cells showed strong positive cytoplasmic staining for Vimentin, C-Kit, CD34 and SMA. Immunohistochemical analysis reported positive focal expression for CD 117 in numerous rhabdoid tumor cells, showing strong cytoplasmic staining and positive focal expression for CD 34 in single tumor cells with large round or fusiform nuclei and in numerous rhabdoid tumor cells of the tumor neoangiogenesis (Figure 5/A,B and Figure $7 / D$ ) and diffuse positive expression for SMA and Vimentin (Figure 5/C,E, Figure 7/A, Figure 8/D). Currently there is some controversy regarding whether a tumor that looks like a GIST on hematoxylin and eosin (H\&E) staining, but that is positive expression for CD 117 in single rhabdoid tumor cells should be called a GIST. The positive IHC expression of spindle tumor cells for SMA and diffuse expression for Viminine represent the rhabdoid cell nature of this GIST subtype (Figure 7/A). Positive expression of spindle tumor cells to SMA distinguishes this malignant tumor from epitheloid angiosarcoma (Figure 5/C and Figure 7/A). Negative expression for cytokeratins (CK 20 and CK AE1-AE3) distinguishes it from non- INS extrarenal rhabdoid tumors, from metastatic undifferentiated carcinoma and epitheloid sarcoma (Figure 6/C,D). Immunohistochemical analysis for Calretinin indicates punctuation accumulation in neurons and has negative expression in tumor cells, which rejects mesothelioma diagnosis (Figure 7/C). Negative expression for the $\mathrm{S}-100$ protein distinguishes it from amelanotic melanoma (Figure 6/F). Negative expression to CDX2 distinguishes this tumor of metastasis from gastrointestinal carcinoma (Figure 6/B).

\section{Complex Treatment}

GISTs should always be removed, since all of these tumors can potentially metastasize. The laparoscopic and open surgical resection procedure with a "gentle-touch technique" is recommended in order to reduce the risk of hemorrhage and intraperitoneal dissemination, as GISTs tend to have a friable consistency [59]. The principal treatment modality for patients with primary GIST is complete surgical resection [26]. A large surgical resection of the tumor (including the total thickness of the gastric wall) or a partial gastrectomy is recommended [60]. $\mathrm{R} 0$ resection (no residual disease) is one of the most important influences for treatment outcome (disease-free interval and survival), and is achieved in around $40 \%$ to $60 \%$ of all cases of GIST and in more than $70 \%$ in cases of non-metastatic disease [61-64]. Nodal metastasis is a rare event, there is no data in the literature indicating the conduction of systematic lymphadenectomy [65]. Lymphadenectomy is not routinely performed in patients with GIST [66,67]. Medical treatment with a tyrosine kinase inhibitor (i.e. imatinib) is indicated for recurrent or iresectable GISTs as this treatment has proven very effective, safe and tolerable [59]. Tyrosine kinase inhibitors (TKIs) (sunitinib and sorafenib) are small-molecular-weight drugs that inhibit the kinase activity of different receptors. The mechanism of action of TKIs relies on binding around the ATP binding site of a given receptor and thus hindering phosphorylation of the tyrosine residue of that receptor and subsequent transmission of signaling down the intercellular pathway [68].

\section{Conclusion}

Rhabdoid signet-ring mixed (spindle and epithelioid) cell subtype GIST is a rare diagnosed extremely aggressive malignant tumor. This is a third rhabdoid histologic phenotype GIST of the stomach posted in the English medical literature [58,69]. Its diagnosis requires an expert pathohistological and immunohistochemical study to determine the differential diagnosis with a number of other malignant mesenchymal tumors. Due to tumor expansion within the muscularis propria of the stomach, endoscopic biopsy is hampered. To allow biopsy in the depth of the stomach muscle layer, endoscopic ultrasound biopsy or laparoscopic biopsy is required. Due to the aggressive malignant potential of the represented rhabdoid phenotype GIST, the prognosis is unfavorable. The initial expression of non-operable distant metastases in parenchymal organs and lymph nodes requires chemotherapy and target therapy. Medical treatment with a tyrosine kinase inhibitor (i.e. Imatinib) is indicated for recurrent or inoperable metastatic GISTs as this treatment has proven very effective.

\section{References}

1. Trupiano JK, Stewart RE, Misick C, Appelman HD, Goldblum JR (2002) Gastric stromal tumors: a clinicopathologic study of 77 cases with correlation of features with nonaggressive and aggressive clinical behaviors. Am J Surg Pathol 26(6): 705-714.

2. Bell P, Findeis-Hosey J (2021) GIST.

3. House MG, Guo M, Efron DT, Lillemoe KD, Cameron JL, et al. (2003) Tumor suppressor gene hypermethylation as a predictor of gastric stromal tumor behavior. J Gastrointest Surg 7(8): 1004-1014. 
4. Mochizuki Y, Kodera Y, Ito S, Yamamura Y, Kanemitsu Y, et al. (2004) Treatment and risk factors for recurrence after curative resection of gastrointestinal stromal tumors of the stomach. World J Surg 28(9): 870-875.

5. Maeda H, Yamagata A, Nishikawa S, Yoshinaga K, Kobayashi S, et al. (1992) Requirement of c-kit for development of intestinal pacemaker system. Development 116(2): 369-375.

6. Robinson TL, Sircar K, Hewlett BR, Chorneyko K, Riddell RH, et al. (2000) Gastrointestinal stromal tumors may originate from a subset of CD34positive interstitial cells of Cajal. Am J Pathol 156(4): 1157-1163.

7. Sanders KM (1996) A case for interstitial cells as pacemakers and mediators of neurotransmission in the gastrointestinal tract. Gastroenterology 111(2): 492-515.

8. Ihab RK, Fishman EK (2006) Chapter "Imaging of Gastrointestinal Stromal Tumor". Oncology pp. 413-424.

9. Fletcher CD, Berman JJ, Corless C, Gorstein F, Lasota J, et al. (2002) Diagnosis of gastrointestinal stromal tumors: a consensus approach. Hum Pathol 33(5): 459-465.

10. Huang XF, Wang CM, Pan BR, Dai XW, Fang L, et al. (2004) Pathologica characteristics of gastric leiomyoblastoma. World J Gastroenterol 10(21): 3182-3184

11. Nassiopoulos K, Stockhammer A, Hahnloser P (1997) [Gastric leiomyoblastoma: literature review and report of a case]. Rev Med Suisse Romande 117(2): 147-150.

12. Linhares E, Gonçalves R, Valadão M, Vilhena B, Daniel Herchenhorn, et al. (2011) Gastrointestinal stromal tumor: analysis of 146 cases of the center of reference of the National Cancer Institute - INCA. Rev Col Bras Cir 38(6): 398-406.

13. La Torre E, Petrozza V, Nicolai AP, Pulvirenti S, Nicastro A, et al. (1996) [Bizarre gastric leiomyoblastoma. A case report and review of the literature]. Minerva Chir 51(4): 235-243.

14. Simeth C, Dellach C, Guarino G, Balani A (1999) [Gastric leiomyoblastoma (Review of the literature in the light of a 15-case)]. Ann Ital Chir 70(1) 57-60.

15. Gonzalez RS (2021) Gastrointestinal stromal tumor.

16. Xiao-Feng H, Chun-Mei W, Bo-Rong P (2004) Pathological characteristics of gastric leiomyoblastoma. World J Gastroenterol 10(21): 3182-3184.

17. Kindblom LG, Remotti HE, Aldenborg F, Meis-Kindblom JM (1998) Gastrointestinal pacemaker cell tumor (GIPACT): Gastrointestinal stromal tumors show phenotypic characteristics of the intestinal cells of Cajal. Am J Pathol 152(5): 1259-1269.

18. Hirota S, Isozaki K, Moriyama Y, Hashimoto K, Nishida T, et al. (1998) Gain-of-function mutations of c-Kit in human gastrointestinal stromal tumors. Science 279(5350): 577-580.

19. Atalay C, Becer M, Ozaslan C (1998) Gastric leiomyoblastoma: report of three cases. Int Surg 83(4): 291-293.

20. Pelandré GL, Djahjah MC, Nobre LF (2008) Aspectos tomograficos do tumor estromal gastrintestinal de origem gástrica: estudo de 14 casos. Radiol bras 41(5): 297-303.

21. Kosmadakis N, Visvardis EE, Kartsaklis P, Tsimara M, Chatziantoniou A, et al. (2005) The role of surgery in the management of gastrointestinal stromal tumors (GISTs) in the era of imatinib mesylate effectiveness. Surg Oncol 14(2): 75-84.

22. Deepa TP, Brian PR (2011) Gastrointestinal Stromal Tumor. Advances in Diagnosis and Management. Arh Pathol Lab Med 135(10): 1298-1309.

23. Greenson J (2003) Gastrointestinal Stromal Tumors and Other Mesenchymal Lesions of the Gut. Mod Pathol 16(4): 366-375.
24. Simmonds N, Furman M, Karanika E (2014) Paneth cell metaplasia in newly diagnosed inflammatory bowel disease in children. BMC Gastroenterol 14: 93.

25. Miettinen M, El-Rifai W, H L Sobin L, Lasota J (2002) Evaluation of malignancy and prognosis of gastrointestinal stromal tumors: a review. Hum Pathol 33(5): 478-483.

26. Ho Gun K, Seong TR, Joo JK, Kang H, Lee JH, et al. (2011) Recurring gastrointestinal stromal tumor with splenic metastasis. J Korean Surg Soc 81(Suppl 1): S25-S29.

27. Rossen NS, Tarp JM, Mathiesen J, Jensen MH, Oddershede LB (2014) Long-range ordered vorticity patterns in living tissue induced by cell division. Nat Commun 5: 5720

28. Gerhardt H, Betsholtz C (2005) Mechanisms of Angiogenesis. In: Clauss M \& Breier G (Eds.), Chapter 8, Birkhauser, Switzerland.

29. Folkman J (1971) Tumor angiogenesis: therapeutic implications. N Engl J Med 285(21): 1182-1186.

30. Welti J, Loges S, Dimmeler S, Carmeliet P (2013) Recent molecular discoveries in angiogenesis and antiangiogenic therapies in cancer. J Clin Invest 123(8): 3190-3200.

31. Bucher P, Taylor S, Villiger P, Morel P, Brundler MA (2004) Are there any prognostic factors for small intestinal stromal tumors? Am J Surg 187(6): 761-766.

32. Roberto de Oliveira das Neves L, Oshima CTF, Artigiani-Neto R (2009) Ki67 and p53 in gastrointestinal stromal tumors - GIST. Arq. Gastroenterol. São Paulo 46(2).

33. Agaimy A (2010) Gastrointestinal stromal tumors (GIST) from risk stratification systems to the new TNM proposal: more questions than answers? A review emphasizing the need for a standardized GIST reporting. Int J Clin Exp Pathol 3(5): 461-471.

34. Demetri GD (2002) Identification and treatment of chemoresistant inoperate or metastatic GIST: experience with the selective tyrosine kinase inhibitor imatinib mesylate (STI571). Eur J Cancer 38(Suppl 5): s52-s59.

35. Miettinen M, Lasota J (2001) Gastrointestinal stromal tumors definition, clinical, histological, immunohistochemical, and molecular genetic features and differential diagnosis. Virchows Arch 438(1): 1-12.

36. Gulwani H (2021) GIST.

37. Blay JY, Bonvalot S, Casali P, Choi H, Debiec-Richter M, et al. (2005) GIST consensus meeting panelists. Consensus meeting for the management of gastrointestinal stromal tumors. Report of the GIST Consensus Conference of 20-21 March 2004, under the auspices of ESMO. Ann Oncol 16(4): 566-578.

38. Miettinen M, Lasota J (2003) Gastrointestinal Stromal Tumors (GISTs): Definition, Occurrence, Pathology, Differential Diagnosis and Molecular Genetics. Pol J Pathol 54(1): 3-24.

39. Carvalho JC, Thomas DG, Lucas DR (2009) Cluster analysis of immunohistochemical markers in leiomyosarcoma delineates specific anatomic and gender subgroups. Cancer 115(18): 4186-4195.

40. Shankar V (2021) Leiomyosarcoma-general. Accessed May 7th, 2021.

41. Domanski HA, Akerman M, Rissler P, Gustafson P (2006) Fine-needle aspiration of soft tissue leiomyosarcoma: an analysis of the most common cytologic findings and the value of ancillary techniques. Diagn Cytopathol 34(9): 597-604.

42. Dixit S, Singhal S, Baboo HA, Vyas RK, Neema JP, et al. (1993) Leiomyosarcoma of the ovary. J Postgrad Med 39(3): 151-153.

43. .Marinova L, Bistra Y, Doroteya M, Kremena P (2020) Pathohistological Diagnosis, Immunohistochemical Analysis and Optimal Complex 
Treatment in Primary Ovarian Leiomyosarcoma. Biomed J Sci \& Tech Res 32(2): 24800-24805.

44. Enzinger FM (1970) Epithelioid sarcoma. A sarcoma simulating a granuloma or a carcinoma. Cancer 26(5): 1029-1041.

45. Armah HB, Anil VP (2009) Epithelioid sarcoma. Archives of Pathology \& Laboratory Medicine 133(5): 814-819.

46. Antonin L, Le Péchoux C, Terrier P (2014) Epithelioid Sarcoma: Need for a Multimodal Approach to Maximize the Chances of Curative Conservative Treatment. Annals of Surgical Oncology 21(1): 269-276.

47. Agafonoff S, Vaidya SK, DeFade B (2016) High-grade undifferentiated pleomorphic sarcoma of the pelvis: A case report and review of literature. Urol Ann 8(3): 372-376

48. Shankar V (2021) Spindle cell/pleomorphic lipoma.

49. Isozaki K, Hirota S, Nakama A, Miyagawa J, Shinomura Y, et al. (1995) Disturbed intestinal movement, bile reflux to the stomach, and deficiency of c-Kit-expressing cells in Ws/Ws mutant rats. Gastroenterology 109(2): 456-464.

50. Hasegawa T, Matsuno Y, Shimoda T, Hirohashi S (2002) Gastrointestinal stromal tumor: consistent CD117 immunostaining for diagnosis, and prognostic classification based on tumor size and MIB-1 grade. Hum Pathol 33(6): 669-676.

51. Miettinen M, Majidi M, Lasota J (2002) Pathology and diagnostic criteria of gastrointestinal stromal tumors (GISTs): a review. Eur J Cancer 38(Suppl 5): s39-s51.

52. Taniguchi M, Nishida T, Hirota S, Isozaki K, Ito T, et al. (1999) Effect of c-KIT mutation on prognosis of gastrointestinal stromal tumors. Cancer Res 59(17): 4297-4300.

53. Heinrich MC, Corless CL, Demetri GD, Blanke CD, Mehren MV, et al. (2003) Kinase mutations and imatinib response in patients with metastatic gastrointestinal stromal tumor. J Clin Oncol 21(23): 4342-4349.

54. Heinrich MC, Corless CL, Duensing A, McGreevey L, Chang-Jie C, et al. (2003) PDGFRA activating mutations in gastrointestinal stromal tumors. Science 299(5607): 708-710.

55. Igwilo OC, Byrne MP, Nguyen KD, Atkinson J (2003) Malignant gastric stromal tumor: unusual metastatic patterns. South Med J 96(5): 512515.

56. Mazur MT, Clark HB (1983) Gastric stromal tumors. Reappraisal of histogenesis. Am J Surg Pathol 7(6): 507-519.

57. Miettinen M, Furlong M, Sarlomo-Rikala M, Burke A, Sobin LH, et al. (2001) Gastrointestinal stromal tumors, intramural leiomyomas, and leiomyosarcomas in the rectum and anus: a clinicopathologic, immunohistochemical, and molecular genetic study of 144 cases. The American Journal of Surgical Pathology 25(9): 1121-1133.

58. Richmond JA, Mount SL (2004) Gastrointestinal Stromal Tumor of the Stomach with Rhabdoid Phenotype: Immunohistochemical, Ultrastructural, and Immunoelectron Microscopic Evaluation. Ultrastructural Pathology 28(3): 165-170.

59. Ponsaing LG, Hansen MB (2007) Therapeutic procedures for submucosal tumors in the gastrointestinal tract. World J Gastroenterol 13(24): 33163322.

60. Nassiopoulos K, Stockhammer A, Hahnloser P (1997) [Gastric leiomyoblastoma: literature review and report of a case]. Rev Med Suisse Romande 117(2): 147-150.

61. Liegl B, Hornick JL, Lazar AJ (2009) Contemporary pathology of gastrointestinal stromal tumors. Hemat Oncol Clin North Am 23(1): 4968.

62. Hassan I, You YN, Shyyan R, Dozois EJ, Smyrk TC, et al. (2008) Surgically managed gastrointestinal stromal tumors: a comparative and prognostic analysis. Ann Surg Oncol 15(1): 52-59.

63. Gutierrez JC, De Oliveira LO, Perez EA, Rocha-Lima C, Livingstone AS, et al. (2007) Optimizing diagnosis, staging and management of gastrointestinal stromal tumors. J Am Coll Surg 205(3): 479-491.

64. Roberts PJ, Eisenberg B (2002) Clinical presentation of gastrointestinal stromal tumors and treatment of operable disease. Eur J Cancer 38(Suppl 5): S37-S38.

65. Casali PG, Jost L, Reichardt P, Schlemmer M, Blay JY, et al. (2008) ESMO Guidelines Working Group. Gastrointestinal stromal tumors: ESMO clinical recommendations for diagnosis, treatment and follow-up. Ann Oncol 19(Suppl 2): ii35-ii38.

66. Kosmadakis N, Visvardis EE, Kartsaklis P, Tsimara M, Chatziantoniou A et al. (2005) The role of surgery in the management of gastrointestinal stromal tumors (GISTs) in the era of imatinib mesylate effectiveness. Surg Oncol 14(2): 75-84.

67. Silberhumer GR, Hufschmid M, Wrba F, Gyoeri G, Schoppmann S, et al (2009) Surgery for gastrointestinal stromal tumors of the stomach. J Gastrointest Surg 13(7): 1213-1219.

68. Ribatti D (2009) History of research on tumor angiogenesis. Springer New York, USA.

69. Lee JR, Joshi V, Griffin JW, Lasota J, Miettinen M, et al. (2001) Gastrointestinal autonomic nerve tumor: immunohistochemical and molecular identity with gastrointestinal stromal tumor. Am J Surg Pathol 25(8): 979-987. 